\title{
THE HEEGAARD GENUS OF AMALGAMATED 3-MANIFOLDS
}

\author{
MARC LACKENBY
}

\section{INTRODUCTION}

When studying Haken 3-manifolds, one is led naturally to the following construction: the amalgamation of two 3-manifolds $M$ and $M^{\prime}$ via a homeomorphism between their boundaries. In this paper, we study the behaviour of Heegaard genus under this operation. We show that, provided the gluing homeomorphism is 'sufficiently complicated' and $M$ and $M^{\prime}$ satisfy some standard conditions, then the Heegaard genus of the amalgamated manifold is completely determined by the Heegaard genus of $M$ and $M^{\prime}$ and the genus of their common boundary. Recall that a 3-manifold is simple if it is compact, orientable, irreducible, atoroidal, acylindrical and has incompressible boundary. We denote the Heegaard genus of a 3-manifold $M$ by $g(M)$.

Main Theorem. Let $M$ and $M^{\prime}$ be simple 3-manifolds, and let $h: \partial M \rightarrow S$ and $h^{\prime}: S \rightarrow \partial M^{\prime}$ be homeomorphisms with some connected surface $S$ of genus at least two. Let $\psi: S \rightarrow S$ be a pseudo-Anosov homeomorphism. Then, provided $|n|$ is sufficiently large,

$$
g\left(M \cup_{h^{\prime} \psi^{n} h} M^{\prime}\right)=g(M)+g\left(M^{\prime}\right)-g(S) .
$$

Furthermore, any minimal genus Heegaard splitting for $M \cup_{h^{\prime} \psi^{n} h} M^{\prime}$ is obtained from splittings of $M$ and $M^{\prime}$ by amalgamation, and hence is weakly reducible.

The amalgamation of two Heegaard splittings, referred to in the above theorem, was defined by Schultens [10]. We recall it here. Since $\partial M$ and $\partial M^{\prime}$ are assumed to be connected, the Heegaard splittings of $M$ and $M^{\prime}$ divide each manifold into a compression body and a handlebody. Each compression body is a copy of $S \times I$ with 1-handles attached. Extend these 1-handles vertically through $S \times I$ so that they are attached to $\partial M$ and $\partial M^{\prime}$ respectively. We may assume that their attaching discs are disjoint when the manifolds are glued. Attach the boundaries of these 1-handles to the copy of $S$ in $M \cup M^{\prime}$, and remove the interiors of the attaching discs. The resulting surface is a Heegaard surface for $M \cup M^{\prime}$, which 
is said to be obtained from the splittings of $M$ and $M^{\prime}$ by amalgamation. (See Figure 1.) By calculating the genus of this surface, we obtain the inequality

$$
g\left(M \cup M^{\prime}\right) \leq g(M)+g\left(M^{\prime}\right)-g(S) .
$$

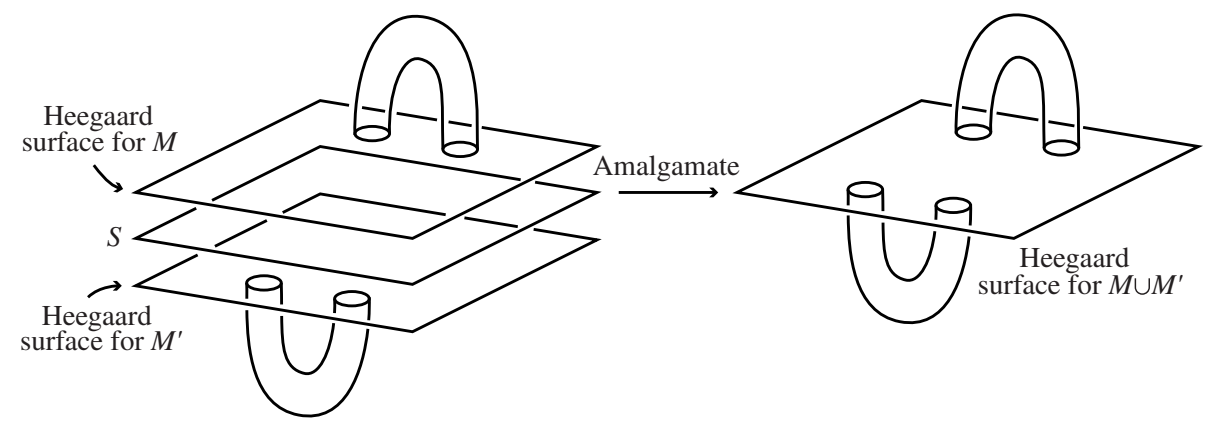

Figure 1.

Inequalities going in the other direction have been discovered by Johannson [3] and Schultens [11] who proved, respectively, that

$$
\begin{aligned}
& g\left(M \cup M^{\prime}\right) \geq \frac{1}{5} g(M)+\frac{1}{5} g\left(M^{\prime}\right)-\frac{2}{5} g(S), \\
& g\left(M \cup M^{\prime}\right) \geq \frac{1}{3} g(M)+\frac{1}{3} g\left(M^{\prime}\right)-\frac{4}{3} g(S)+\frac{5}{3} .
\end{aligned}
$$

Clearly, in general, one will not be able to determine $g\left(M \cup M^{\prime}\right)$ precisely in terms of $g(M), g\left(M^{\prime}\right)$ and $g(S)$. Inequalities such as the above will have to suffice. It is therefore slightly surprising that, for complicated gluings, an exact formula as in the main theorem should hold.

\section{Proof of the MAIN THEOREM}

The amalgamated manifold $M \cup M^{\prime}$ is Haken, atoroidal and not Seifert fibred. So, by Thurston's geometrisation theorem, it admits a hyperbolic structure. In [12], Soma gave a careful analysis of its geometry. Soma proved that one can find a point $x_{n}$ in each $M \cup_{h^{\prime} \psi^{n} h} M^{\prime}$ such that the based manifolds $\left(M \cup_{h^{\prime} \psi^{n} h} M^{\prime}, x_{n}\right)$ converge in the Gromov-Hausdorff topology to the infinite cyclic cover of the hyperbolic fibred 3-manifold with monodromy $\psi$. Furthermore, any fibre in the limit space pulls back to a surface isotopic to the copy of $S$ in $M \cup_{h^{\prime} \psi^{n} h} M^{\prime}$, provided $|n|$ is sufficiently large. Hence, we deduce that, provided $|n|$ is sufficiently large, one may find in $M \cup_{h^{\prime} \psi^{n} h} M^{\prime}$ an arbitrarily large number of parallel copies 
of $S$, such that any two adjacent copies have distance at least one from each other. We denote the product region in $M \cup_{h^{\prime} \psi^{n} h} M^{\prime}$ between the extreme copies of $S$ by $S \times I$. Then we may also ensure that there is an $\epsilon>0$, independent of $n$, such that $S \times I$ lies in the $\epsilon$-thick part of $M \cup_{h^{\prime} \psi^{n} h} M^{\prime}$.

Now consider a minimal genus Heegaard surface $F$ for $M \cup M^{\prime}$. Note that $g(F) \leq g(M)+g\left(M^{\prime}\right)-g(S)$. From $F$, we construct (as in [7], [8] or [4]) a generalised Heegaard splitting $\left\{F_{1}, \ldots, F_{m}\right\}$ with the following properties:

- $F_{j}$ is strongly irreducible, for each odd $j$;

- $F_{j}$ is incompressible and has no 2-sphere components, for each even $j$;

- $F_{j}$ and $F_{j+1}$ are not parallel for any $j$;

- $\sum_{j}(-1)^{j} \chi\left(F_{j}\right)=-\chi(F)$;

- $\left|\chi\left(F_{j}\right)\right| \leq|\chi(F)|$ for each $j$.

Let $F_{+}=F_{1} \cup \ldots \cup F_{m}$. The third and fourth conditions imply that $m \leq|\chi(F)|$, and hence the fifth gives that $\left|\chi\left(F_{+}\right)\right| \leq|\chi(F)|^{2}$, a bound which is independent of $n$. One can obtain $F$ back from $F_{+}$by amalgamating $F_{1}$ and $F_{3}$, then amalgamating this with $F_{5}$, and so on.

By theorems of Schoen and Yau [9], Freedman, Hass and Scott [1] and Pitts and Rubinstein [5], each component of $F_{+}$may be isotoped to a minimal surface or to the double cover of a minimal non-orientable surface (possibly with a small tube attached in the case of an odd surface). Furthermore, after these isotopies, any two components are either equal or disjoint. Each complementary region of $F_{+}$after the isotopies corresponds to one before, but some product complementary regions may have been collapsed. In particular, each complementary region afterwards is a compression body.

We would like to apply Proposition 6.1 of [4], which gives a constant $k$, such that each component $F^{\prime}$ of $F_{+}$has diameter at most $k\left|\chi\left(F^{\prime}\right)\right|$. (Here, we are using the path metric on $F_{+}$arising from its induced Riemannian metric.) However, $k$ depends on a positive lower bound for the injectivity radius of the ambient manifold. It is not immediately clear from Soma's paper whether there is such a bound that is independent of $n$. We will therefore present a variant of Proposition 
6.1 of [4]. Let $\delta$ be $2 \epsilon+1+\epsilon / \pi$. We claim that we can cover $F_{+} \cap(S \times I)$ with regions, each of which has diameter at most $\delta$ in $F_{+}$, and so that the total number of regions is at most $k\left|\chi\left(F_{+}\right)\right|$, for some constant $k$ independent of $n$. These regions will be of two types.

Let $\left(F_{+}\right)_{[\epsilon, \infty)}$ and $\left(F_{+}\right)_{(0, \epsilon]}$ be the $\epsilon$-thick and $\epsilon$-thin parts of $F_{+}$. Let $\Gamma$ be a maximal collection of disjoint (not necessarily simple) closed geodesics in $F_{+}$, each with length less than $\epsilon$. The first type of region will consist of those points within $\epsilon / 2+\epsilon /(2 \pi)+1 / 2$ of some component of $\Gamma$. Clearly, each such region has diameter at most $\delta$. Claim 3 in the proof of Proposition 6.1 of [4] gives that there are at most $4\left|\chi\left(F_{+}\right)\right|$geodesics in $\Gamma$, and hence at most $4\left|\chi\left(F_{+}\right)\right|$such regions. The argument of Claims 2 and 1 there also gives that these regions cover $\left(F_{+}\right)_{(0, \epsilon]} \cap(S \times I)$. This uses the assumption that $S \times I$ lies in the $\epsilon$-thick part of $M \cup M^{\prime}$.

Now pick a maximal collection of points in $\left(F_{+}\right)_{[\epsilon, \infty)} \cap(S \times I)$, no two of which are less than $\epsilon$ apart in $F_{+}$. Then the $\epsilon$-balls around these points cover $\left(F_{+}\right)_{[\epsilon, \infty)} \cap(S \times I)$, and there are at most $(\cosh (\epsilon / 2)-1)^{-1}\left|\chi\left(F_{+}\right)\right|$such balls. Letting these be the other type of region, we have established the claim.

We claim that one of the parallel copies of $S$ is disjoint from $F_{+}$, when $|n|$ is sufficiently large. Since each of the regions into which we have divided $F_{+} \cap(S \times I)$ has uniformly bounded diameter, there is a uniform upper bound on the number of copies of $S$ it can intersect. There is also a uniform upper bound on the number of such regions. Hence, there is a uniform upper bound on the number of copies of $S$ that $F_{+}$can intersect. When $|n|$ is sufficiently large, there are more copies of $S$ than this bound. This proves the claim. (See Figure 2.)

So, some copy of $S$ lies in the complement of $F_{+}$, which is a collection of compression bodies. Since $S$ is incompressible, it must be parallel to a component of $F_{j}$ for some even $j$. Thus, if we were to cut $M \cup M^{\prime}$ along $S$, we would obtain generalised Heegaard splittings for $M$ and $M^{\prime}$. Amalgamate each of these, to form Heegaard surfaces $\tilde{F}$ and $\tilde{F}^{\prime}$ for $M$ and $M^{\prime}$. Then, $F$ is obtained by amalgamating $\tilde{F}$ and $\tilde{F}^{\prime}$ along $S$.

This implies that $g(F)=g(\tilde{F})+g\left(\tilde{F}^{\prime}\right)-g(S) \geq g(M)+g\left(M^{\prime}\right)-g(S)$. Since we already have the opposite inequality, the theorem is proved. 


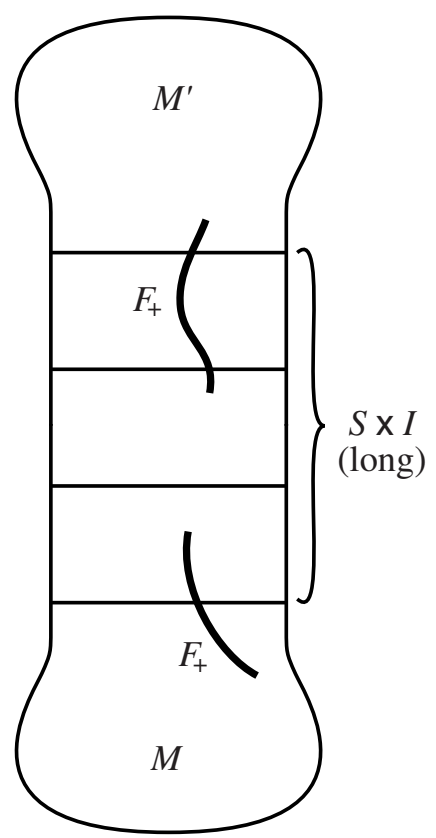

Figure 2.

\section{Generalisations}

The main theorem is not the most general possible statement one can make. In fact, the proof gives the following stronger result.

Theorem. Let $M, M^{\prime}, S, h, h^{\prime}$ and $\psi$ be as in the main theorem. Then for each $g>0$, there is an $N>0$ with the following property: if $|n| \geq N$, then any genus $g$ splitting for $M \cup_{h^{\prime} \psi^{n} h} M^{\prime}$ is obtained from splittings of $M$ and $M^{\prime}$ by amalgamation. In particular, it is weakly reducible.

There is a related way of building a Haken 3-manifold via gluing: one can start with a single simple 3-manifold $M$, and glue two of its boundary components via an orientation-reversing homeomorphism. In this case, we obtain a similar result to the main theorem, but do not obtain a precise equality.

Theorem. Let $M$ be a simple 3-manifold, and let $Y$ and $Y^{\prime}$ be distinct boundary components of $M$. Suppose that there is an orientation-preserving homeomorphism $h: Y \rightarrow S$ and an orientation-reversing homeomorphism $h^{\prime}: S \rightarrow Y^{\prime}$, where $S$ is some surface of genus at least two. Let $\psi: S \rightarrow S$ be a pseudo-Anosov homeomorphism, and let $M / \sim$ be the manifold obtained by gluing $Y$ and $Y^{\prime}$ via $h^{\prime} \psi^{n} h$. 
Then, provided $|n|$ is sufficiently large,

$$
g(M)-g(S)+1 \leq g(M / \sim) \leq g(M)+g(S)+1 .
$$

The proof is very similar, but not identical, to that of the main theorem. To achieve the upper bound on $g(M / \sim)$, one starts with a minimal genus splitting for $M$, and uses it to construct a splitting for $M / \sim$. One might have to modify the surface in $M$ to ensure that it does not separate $Y$ from $Y^{\prime}$. This may increase its genus by $g(S)$. Then, to construct a Heegaard surface for $M / \sim$, one attaches a tube that runs through $S$. This increases the genus of the surface by one. Hence, we obtain the upper bound. An instructive example is where $M$ is the product $S \times I$ of a closed orientable surface and an interval, and where $M / \sim$ fibres over the circle. (Of course, though, $M$ is not simple in this case.) Then, $g(M)=g(S)$, but in general, $g(M / \sim)$ may be as much as $2 g(S)+1$. (See [6] for example).

To achieve the lower bound on $g(M / \sim)$, one starts with a minimal genus Heegaard surface $F$ for $M / \sim$. One untelescopes it to a generalised Heegaard splitting satisfying the five conditions given earlier. Using the geometry of $M / \sim$, one can show that this is disjoint from a copy of $S$ in $M / \sim$, provided $|n|$ is sufficiently large. Thus, it gives a generalised Heegaard splitting for $M$, which can be amalgamated to form a Heegaard surface. One calculates its genus to be $g(F)+g(S)-1$.

The same issues arise when gluing simple manifolds $M$ and $M^{\prime}$ but when $\partial M$ and $\partial M^{\prime}$ are disconnected. Again, one does not obtain an exact equality.

It should be possible to generalise the main theorem even further. One can consider the manifold $M \cup_{h^{\prime} \psi h} M^{\prime}$, where $\psi: S \rightarrow S$ is some homeomorphism. It should be true that, under the hypotheses of the main theorem, and provided the distance of $\psi$ is sufficiently large, then the conclusion of the main theorem holds. Here, distance is as measured by the action of $\psi$ on the curve complex of $S$. This would indeed represent a generalisation, since the distance of $\psi^{n}$, for a given pseudo-Anosov $\psi$, is arbitrarily large, provided $|n|$ is sufficiently large [2]. One might also try to drop the assumptions that $M$ and $M^{\prime}$ are acylindrical, or even that they have incompressible boundary. But one would then need to make further hypotheses on $\psi$. To prove these more general results, one would need to establish geometric control on $M \cup M^{\prime}$, using the theory of Kleinian groups. 


\section{REFERENCES}

1. M. Freedman, J. Hass And P. Scott, Least area incompressible surfaces in 3-manifolds, Invent. Math. 71 (1983) 609-642.

2. J. Hempel, 3-manifolds as viewed from the curve complex, Topology $\mathbf{4 0}$ (2001) 631-657.

3. K. Johannson, Topology and combinatorics of 3-manifolds, Springer-Verlag (1995).

4. M. LaCKenBy, Heegaard splittings, the virtually Haken conjecture and Prop$\operatorname{erty}(\tau)$, Preprint.

5. J. Pitts And J. H. Rubinstein, Existence of minimal surfaces of bounded topological type in three-manifolds. Miniconference on geometry and partial differential equations (Canberra, 1985), 163-176.

6. J. H. Rubinstein, Minimal surfaces in geometric 3-manifolds, Preprint.

7. M. Scharlemann, Heegaard splittings of compact 3-manifolds, Handbook of Geometric Topology, Elsevier (2002), 921-953.

8. M. Scharlemann and A. Thompson, Thin position for 3-manifolds, Geometric topology (Haifa, 1992), 231-238, Contemp. Math., 164.

9. R. Schoen and S. Yau, Existence of incompressible surfaces and the topology of 3-manifolds with non-negative scalar curvature, Ann. Math. 110 (1979) 127-142.

10. J. Schultens, The classification of Heegaard splittings for (compact orientable surface) $\times S^{1}$, Proc. London Math. Soc. 67 (1993), 425-448.

11. J. Schultens, Heegaard genus formula for Haken manifolds, Preprint.

12. T. Soma, Volume of hyperbolic 3-manifolds with iterated pseudo-Anosov amalgamations, Geom. Ded. 90 (2002), 183-200.

Mathematical Institute, Oxford University,

24-29 St Giles', Oxford OX1 3LB, United Kingdom. 